\title{
Response-Ability: Practicing Integrity Through Intimacy in the Marketplace
}

\author{
Kyoko Fukukawa ${ }^{1}$
}

Received: 7 February 2017 / Accepted: 24 April 2018 / Published online: 30 April 2018

(c) The Author(s) 2018

\begin{abstract}
The paper addresses the problem of pursuing ethical business practices purely under the aegis of 'integrity', as frequently used to characterise morally desirable traits. Drawing on the work of philosopher Thomas Kasulis, the paper pairs 'integrity' with 'intimacy' as a critical concept, placing greater attention upon relational properties, helping to understand ethics as existing between individuals, things and the environment. The argument is that by paying careful attention to spatial and temporal dynamics and proximities of exchange, businesses can better maintain and extend practices of integrity. It reminds us that ethics are developmental (not transcendental); that the cultivation of ethics provides greater depth and ownership and pertains to matters of the body and habits. The paper contributes a way of reading exchanges in the marketplace beyond prescriptive accounts of integrity. Through the lens of both integrity and intimacy, it identifies how we actually 'live' or practice greater responsiveness to exchanges.
\end{abstract}

Keywords Integrity $\cdot$ Intimacy $\cdot$ Responsiveness

\section{Response-Ability: Practicing Integrity Through Intimacy in the Marketplace}

In thinking through an ethics of both integrity and intimacy, this paper argues for a more nuanced reading of how 'responsible' exchanges take place in the everyday marketplace. Of particular pertinence is the way in which individuals interact in the service domain. ${ }^{1}$ As Audi and Murphy (2006, p. 3) note, integrity is a key topic in business ethics, 'quite possibly the most commonly cited morally desirable trait'. In the business context, integrity is commonly understood in terms of responsible management and leadership (Bauman 2013). Yet, historically this term has lacked clear articulation, and furthermore it can often signify 'a trait that is good in itself without constituting a moral virtue' (Audi and Murphy 2006, pp. 12-13). Rather than examining the higher-level rhetoric and company strategies of integrity, this paper situates the term at the level of person-to-person interaction, relating to sites of everyday market exchange.

Kyoko Fukukawa

k.fukukawa@bradford.ac.uk

1 School of Management, University of Bradford, Emm Lane, Bradford, West Yorkshire BD9 4JL, UK
In doing so, it draws attention to a more phenomenological and quotidian experience of market exchange.

The paper draws specifically upon Kasulis' (2002) account of integrity and intimacy, with the latter referring to 'an inseparability, a belonging together, a sharing' (p. 24), involving, for example, the sharing of tacit knowledge and greater awareness of situations and of the connection between one and another. Importantly, this is not the intimacy of lovers, but rather underlying structures (or we might say the medium) of exchange between people. 'We speak of the intimate relation between flora and fauna in a particular ecosystem, for example, or between matter and energy in the context of particle physics. In such cases, we cannot fully understand one side of the pair without consideration of the other' (Kasulis 2002, p. 28). However, despite describing a relationship between intimacy and integrity, Kasulis tends to set the two terms in contrast (often presenting them with the grammatical operator 'or' not 'and'). This paper draws on the insights of his work, but seeks to extend the account in two ways. Firstly, it explores intimacy in relation to integrity specifically in the context of business

\footnotetext{
${ }_{1}$ The analysis of the paper focuses upon services, rather than goods, although as the literatures of 'service-dominant logic' suggest, even goods are largely marketed through the means of services (Vargo and Lusch 2008).
} 
and business ethics. In this sense, it applies Kasulis' work in a new context. Secondly, the paper seeks to put forward the potential of an Integrity-Intimacy approach, to suggest of a critical, dialectical approach, which ideally can lead to further research initiatives and also impact on practical business endeavours, such as employee training and the design of customer settings (as referenced in the latter half of this paper, and outlined in implications).

In his book Intimacy or Integrity (2002), Kasulis presents what he calls a 'cultural philosophy'. He is interested in how we think about the relations among people and things according to cultural backgrounds. However, importantly, his arguments move beyond cultural relativism. He is interested in patterns of relations that construct cultures (in this respect his work can be made to connect with Bourdieu's critical term habitus, as will be discussed later, through which we can analyse a range of settings, discourses and structures of exchange). These patterns can alter and scale according to numerous factors and are certainly not fixed to national cultures. Thus, Kasulis' work can readily be applied to specific situations, such as business and/or consumer cultures, as attempted here. In seeking to put forward an IntegrityIntimacy approach, the paper picks up particularly on the philosophical prompt that 'intimacy' can be made observable. The paper extends this point, to allow the empirical nature of intimacy (within the context of the marketplace) to be made more explicit, which in turn opens up future agendas for training and management of business ethics more broadly. Crucially, it is an account that can help us to consider market exchanges as defined not by a single individual or site of origin, but rather through relational means, which in turn enables us to reappraise ethics in the context of the marketplace.

The paper can also be said to echo Robert Solomon (1992, p. 111) when he suggests we 'defend business ethics as a more personally oriented ethics rather than as public policy, "applied" abstract philosophy'. Importantly, again to echo Solomon, the paper does not seek to be drawn into gendered readings of 'care ethics', whereby a 'sharp contrast' is drawn 'between the good warm, feminine virtues of caring and concern and the oppressive, impersonal, war-mongering masculine principles of justice and duty' (p. 116). Instead, the point is to consider a more underlying, sharable array of characteristics and capabilities that we might cultivate. The point, then, is not to conceive of business ethics as 'personal' or 'subjective', but rather to identify a 'social and institutional self-awareness, a sense of oneself as an intimate (but not inseparable) part of the business world with a keen sense of the virtues and values of that world' (Solomon 1992, p. 111). As a contribution to business ethics, this paper offers a way of reading various exchanges in the marketplace that go beyond prescriptive accounts of integrity, and instead, through the lens of both integrity and intimacy, identifies how, together, we actually 'live' or practice matters of integrity. Crucially, intimacy is to be understood as a subtle, yet observable set of qualities, the development of which has the potential to enable businesses to better understand the nature and quality of their engagements with stakeholders, with the particular focus in this paper on the relationship between provider and consumer.

Intimacy equates to greater attentiveness, hence helping in being more responsive to a situation and to anticipate what otherwise might be left unattended. ${ }^{2}$ Importantly, adopting the term 'intimacy' helps temper how we read 'integrity', to reveal an inherently social context, which involves more than merely possessing a sense of what is one's responsibility. It requires the ability to be responsive to others and to wider circumstances. The modernist composer, John Cage (1957, p. 10), once referred to the word 'responsibility' as 'response ability', shifting the emphasis from an ethics of accountability to an aesthetics of engagement. ${ }^{3}$ Similarly, the emphasis here is on practical sensations and responses that arise in given situations. In this respect, references in this paper to 'responsiveness' can be understood as being different to more strategy-based accounts, whereby, for example, we might refer to a company being responsive to its customers perhaps by responding directly to customer feedback etc. Selznick (1994) has written on 'responsiveness enterprise', to suggest of an 'inner commitment to moral restraint', that goes beyond accountability, or being held responsible (p. 345). He is concerned, for example, with how we might be 'genuinely other-regarding' (which, for example, draws in consideration of Buddhist accounts of the self). However, reference to an 'inner' quality leads back to integrity based upon specific sites of agency and autonomy. The account is analytical, which remains somewhat abstract (certainly when compared with the account suggested here through reference to intimacies). The paper is certainly sympathetic to these

\footnotetext{
2 The paper is sympathetic to arguments made by Painter-Morland (2011), who breaks from traditional ways of thinking about 'responsibility' in terms of character traits and fixed notions of identity and agency. She argues a different ontological account whereby 'agency emerges as a side-product of our human pursuits', leading us to need to 'redefine responsibility as a commitment to ongoing responsiveness to changing environments, rather than as some fixed role or trait' (p. 84). The account offered here shares the view that responsibility equates to 'more fluid relational dynamics that emerge between individuals, groups and institutions' (Painter-Morland 2011, p. 91). However, in applying Kasulis's account of 'intimacy', the paper remains attuned to epistemological and empirical matters, rather than presenting an ontological account.

3 Aesthetics here is not to be taken as simply the study of beauty (a meaning that develops only in the eighteenth and nineteenth centuries in Germany and England respectively), but the more fundamental meaning of our perception by the senses, as we get from the Greek term aisthētikos, which is from aisthēta, meaning 'perceptible things'. What can be included, then, is not merely an ability to see or hear phenomena, but that which relates to emotions and sensations.
} 
various arguments and framings, but by invoking an aesthetics of ethics (through the reference to Cage), responsiveness here pertains more specifically to the body, environment, individual and group rhythms, and other associated elements of exchange. A distinction is evoked later on between being responsive 'to' and being responsive 'with'. The former relates more to logical and legalistic forms of exchange. The latter, with which this paper more readily aligns, relates to broader questions and situations of 'being with' others, and responding according to a variety of prompts and rhythms that occur through arrangements with others.

Conceptually, a parallel might be drawn with work of the philosopher Jacques Rancière (2004), who is frequently cited in debates of the 'political aesthetic'-referring not to the aestheticization of politics (such as we might think of with propaganda or heightened symbolism etc.), but rather the underlying fact that all politics is mediated in some fashion, that there is always a 'sense' of politics. This paper is concerned with an ethics not a politics, but similarly with the micro consideration of what underlies its account of responsibility and responsiveness. As will be discussed, intimacy can be considered according to various aspects, positioned around ideas of proximity, anticipation and improvisation. Subsequently, there are practical implications for how firms seek to conduct their day-to-day business and how they might choose to invest in and develop their operations (e.g. through training and relationship management). In short, it is important not only to know who one's stakeholders are, but also how to be responsive towards them.

\section{Intimacies of Integrity}

Dating back at least to Adam Smith's account of the 'invisible hand' and leading on through the debates epitomised by the positions of Friedman (of the-corporate-responsibility to maximise profits) and Freeman (for the wider needs of stakeholders), critical considerations have long been made regarding the 'responsibilities' of companies. Notably, as Painter-Morland (2011) suggests, over the past 25 years, 'the stakeholder perspective won out over a single-minded concern for shareholder profit', yet nonetheless, while it has been established that 'most corporations do have an interest in being responsible towards a broad range of stakeholders ... many other issues remain unresolved' (p. 84). PainterMorland argues for a relational ontology of agency, which destablises our ability to define who acts or makes a decision to cause benefit/harm to stakeholders. Pertinent to such a view, Kasulis' (2002) use of the terms 'integrity' and 'intimacy' can be described respectively (and in brief) as the difference between being 'responsible' and 'responsive'. Crucially, his pragmatic philosophy helps re-cast the reading we might make with existing literatures on responsiveness (as with the caveat noted above with respect to this paper's particularly framing of responsiveness). Different to PainterMorland's (2011) insightful, yet esoteric account of 'responsiveness', Kasulis can be adapted to open up more practical implications for future research in the business context, including analysis of spatial and temporal arrangements of the consumer experience. His cultural philosophy helps prompt a different or supplementary reading to the dominant ethical philosophies associated with debates of integrity and responsibility within the business context.

A good deal of literatures concerned with integrity in management focus upon defining integrity in a normative sense and typically refer to integrity as a matter of leadership, and so consider how integrity is 'transmitted' through company practices, rather than as being relational. For Kasulis, integrity equates to an adherence to codes and rules, which inevitably are established in some way. We can view this a form of transmission model, i.e. that integrity can emanate from a point of origin and be measured accordingly. Culturally, however, points of view can vary substantially. Kasulis notes how the English word for 'rights' is clearly rendered in Japanese, as 'kenri'. In the first instance, from a universalist perspective. We may view this a shared concept. Yet, the term itself is used with vastly different frequency (and significance) in either the English-speaking or Japanese-speaking contexts (cf. Fukukawa and Teramoto 2009, on jinken [human rights]). 'The transfer of words or even ideas', Kasulis notes, 'from one culture to the next does not entail a shared cultural view of what is important' (p. 6). It is important to stress, however, this is not to present an essentialist account. The argument is that different cultural contexts give rise to differing points of emphasis and conduct, but that these are not culturally fixed.

The more interesting analysis comes at the 'subcultural' level-which, as will be developed later in the paper, we can equate with Bourdieu's notion of habitus-i.e. that individuals relate to the shared, social world based upon a system of embodied, habitual dispositions and tendencies, typically shared among those with similar backgrounds (Lizardo 2004). Kasulis tells, for example, of how a doctor and a nurse respond to a patient's pain in different ways, based upon their different types of training and professional roles. The doctor's response is more associated with 'integrity', whereby she upholds her duty according to a set of rules and guidelines. She tells the patient that medication to ease the pain is not due for a set number of hours and nothing more could be done (keeping in mind the need to inhibit the likelihood of dependency). The nurse later reassures the patient that if the night proves difficult again he could ring for the on-call doctor, who may be a little more flexible than the consultant. For Kasulis, the nurse (and potentially the oncall doctor) represent a more intimate, situational approach. This example quickly illustrates one definition of integrity 
and intimacy. Yet, it is problematic in suggesting too great a contrast, with integrity simply the upholding of rules, and intimacy the responding to a person's individual situation (as if to bend the rules). Whereas, in fact, both cases involve a relationship between individuals as they negotiate differing matters of integrity. The view of this paper is that integrity and intimacy are more intricately involved.

The difference implied, however, is the degree to which the patient is acknowledged and brought into the conversation and decision-making. Intimacy can be understood to identify a form of mediation or 'collaboration' between parties involved. Thus, rather than make specific judgements about integrity, this paper is concerned with how integrity is upheld - how it comes to be a function of a given moment in exchange. The introduction of the term 'intimacy' is to acknowledge and examine subtle instances of exchange. It is also to signify certain somatic qualities that relate to phenomenological and embodied forms of knowledge. Kasulis explains, for example, how intimate knowledge 'is achieved through praxis, and it is embodied through physical style' (2002, p. 47). He gives an example of chopping wood with an axe, noting how bodily movement and the design of the axe become a single function. We come to learn through doing, 'the intimacy deepens as the praxis is repeated and habitualized' (p. 43). The significance of which is that through praxis we reach an understanding or way of responding, and in a manner that is not achievable through theorization. In fact, one methodological difficulty is that as soon as we come away from the situation we arguably loose the 'object' of analysis; just as when we put the axe down, it no longer possesses its function as something that swings down and cuts. Of course, added to this is also the complexity of the term integrity, which is considered below across its different meanings before being situated within the terms of intimacy, so as to combine both integrity-intimacy as an underlying practice.

While the word 'integrity' is far from straightforward, it is frequently used with respect to ideal personal characteristics of professionals (both of managers and employees). Monga (2016) notes two streams of research: One is 'the objective view which defines integrity as a morally and ethically neutral term'; another is 'a normative view which explicitly include morality and ethics' (Monga 2016, p. 415). Within the objectivist view, integrity is frequently defined in terms of 'wholeness', as, for example, 'a state or condition of being whole, complete, unimpaired, unbroken, sound, in perfect condition' (Jensen 2009, p. 18). In the business context, 'wholeness' often underlines descriptions of an individual or company's characteristics that are thought to be coherent. A high degree of alignment between a company's mission, strategic planning and operations, for example, could be considered an approach of integrity. Similarly, an individual manager who aligns actions with a consistent set of ideals can often be described as a person of integrity. Empirical investigation that takes an objectivist view focuses on completeness and consistency between word and action because it is assumed that 'honoring your word is an actionable pathway to creating whole and complete social and working relationships through being trusted by others' (Monga 2016, p. 416). Within the leadership literature this view is labelled 'behavioural integrity' (Bauman 2013). However, such an approach has been 'criticised because this concept of integrity can be applied to both a tyrant as well as an ethical person' (Monga 2016, p. 416; see also; Bauman 2013; Audi and Murphy 2006).

Alternatively, the meaning of integrity within the normative view explicitly includes morality and ethics (Monga 2016, p. 415), and is labelled 'moral integrity' within leadership literature, which is to 'preclude immoral or amoral ideals and values' (Krylova et al. 2017, p. 197). In this case 'integrity is presumed to be linked to numerous valuesrelated constructs including ethics, morality, honesty, and sincerity' (p. 196). Inevitably, there are different accounts, with a notable 'divergence of definitions of the concept of integrity' (p. 196). Audi and Murphy (2006), for example, examine a variety of definitions of integrity which resonate with various moral characters (see Paine 1997; Solomon 1992; Dalla Costa 1998; Gostick and Telford 2003; Zauderer 1992, pp. 27-28; DeGeorge 1993). Given many different definitions and perspectives, it is difficult to find a unifying approach. Maak's (2008) seven necessary conditions (commitment, conduct, context, consistency, coherence and continuity) are, according to Monga (2016, pp. 418-419), an antecedent framework. Similarly, Bauman's (2013, p. 422) account of what he terms 'substantive leadership integrity' seeks to define underlying characteristics. Both Maak and Bauman place importance on the idea of 'commitment'. Bauman writes, for example, of needing to have 'identityconferring commitments to values' (p. 422). Along similar lines, Monga (2016, p. 419) argues, 'at the heart of integrity is commitment and adherence to the sound moral principles'. An underlying problem with these accounts - and the way in which commitment of an individual is emphasised-is that they are suggestive of a transmission of integrity, rather than of a set of relations. In other words, integrity is seemingly borne of a singular account or upholding of certain values, which must be pushed through all activities of a business, so suggestive of a top-down approach, arguably with little opportunity to relating integrity to situational (and everyday) circumstances. This is particularly the case where integrity is the subject of literatures on leadership, and which contrasts with critical management literatures (e.g. PainterMorland 2011).

Audi and Murphy (2006, p. 9) go some way to recognise the problem, which stems from the more common reading of integrity as being integral to a person (or a company). The 
logic, they note, is that 'if honesty is integral to a person, a person of integrity cannot normally deliberate about whether or not to be honest'. Yet, in reality, 'achieving fairness and balance may require deliberation and tradeoffs among conflicting demands' (Audi and Murphy 2006, p. 9). Alternatively, then, Audi and Murphy argue we view integrity as integration - as in the integration of, or between elements of characteristics and/or virtues. They distinguish between two kinds of virtue: Substantive moral virtues (e.g. honesty, fairness), which are traits with 'morally good' connotations; and 'adjunctive' virtues (e.g. courage), which do not immediately present a sense of moral sentiment. The argument is that integrity (as integration) falls into this second category of virtue as adjunctive rather substantive. Courage and conscientiousness, for example, are not morally good in themselves, yet they provide a means or a form of mediation for other moral qualities. As Audi and Murphy put it: 'A structure of bricks will not be strong without cement; this does not entail that cement is a building block' (p. 12). This metaphorical reference to 'cement' (as much as the 'building blocks') is of particular pertinence to this paper. The aim - in the context of the marketplace — of bridging between notions of integrity and intimacy (in extending Kasulis' account) is to inquire closely into the 'cement', into how we behave with integrity (rather than seek to pin down simply what we mean by integrity). In other words, how do we understand the cement as binding agent or medium that enables us to engage in a situation with integrity? Such a question opens up consideration of how we can empirically approach integrity as a 'body' of living gestures and exchanges.

In looking more to the 'medium' or mediation of integrity, we need to frame things in a more transactional sense. In other words integrity cannot be defined as a static state, but always a means of negotiating or integrating between states. Such an account is made in Cunliffe and Eriksen's (2011) article on 'Relational Leadership'. They argue, for example, that "Responsibility" is not just something that is formalized in job descriptions, directives and policy documents, but is fundamental to, and situated in, everyday relationships' (2011, p. 1439). However, the emphasis here is of a more integrated, transactional basis. Cunliffe and Eriksen refer to 'relational integrity' (2011, p. 1439), which is concerned not just with establishing principles among a group, but with the very context in which such principles emerge and must be sustained. The idea of situating matters of integrity in the marketplace (indeed in any form of commercial exchange) is pertinent, taking us a step further toward intimacies of exchange.

Audi and Murphy (2006, p. 13) note, aesthetic sensitivity, imagination, and other such abilities, while good in themselves, are not moral virtues. Yet, equally, moral virtues do not govern conduct in isolation from other traits. By exploring ethics from the perspective of the adjunctive-of integrity as integration-we turn from merely logical prescriptions of moral content to questions of form, medium and aesthetics. It is to consider the grounds of ethicality, rather than simply ethical statements. It is at this level that introducing the term 'intimacy' is aimed at focusing on how we personally and concretely attend to matters of integrity.

Working towards an account of both integrity and intimacy involves new levels of description; to reflect upon the aesthetics or means of exchange. In doing so we can attend to the micro setting, a reminder that "management is mainly "people dealing with people"-rather than abstract problem solving' (Solomon 1992, p. 117). Responsibilities remain at the level of the individual, Solomon argues, but importantly the individual understood within a social setting.

The ethical problems that the average manager faces on the job are personnel and routine administrative decision-making problems, not policy problems. Some of those problems have to do with temptations [...] Some have to do with conflicts of duties, mixed messages, crossed loyalties. [...] Whatever else business ethics may involve and however sophisticated its theories may become, it means knowing that even such decisions (and their consequences) are nevertheless one's own to live with. Ethics is not just a subject for executive boards, planning committees, and government overseers but for all of us, in the details as well as the larger dramas of our everyday lives. (Solomon 1992, pp. 112-113)

Solomon's Aristolelean account of business ethics, which foregrounds the everyday and our personal fallible engagements, seeks to avoid 'abstract role-transcendent morality'. It is to position against the main rationalist accounts of ethics (i.e. against deontological or duty-defined ethics associated with Kant, or utilitarian theory associated with John Stuart Mill). ${ }^{4}$ By contrast, Aristotle takes a starting point from community and context, arguing that it is cultivation of personal character that counts, long before we begin to rationalize our actions' (Solomon 1992, pp. 113-114). In

\footnotetext{
${ }^{4}$ It is worth noting, Solomon's (1992, pp. 115-117) account of Aristotle's 'business ethics' leads to a form of 'virtue ethics', but which he positions carefully. He does not wish to be drawn into certain debates about 'tradition' or 'community' as nostalgic terms, nor does he accept certain gendered readings, "which have drawn a sharp contrast between the good warm, feminine virtues of caring and concern and the oppressive, impersonal, war-mongering masculine principles of justice and duty' (p. 116). Instead, he draws attention for the need of a broader array of characteristics and capabilities that we might cultivate. Intimacy we might suppose is one such virtue worth cultivating. However, before turning directly to this concept and its interaction with matters of integrity, it is first pertinent to identify what is meant by integrity in the context of business and the marketplace.
} 
what will be discussed subsequently in terms of practicing intimacy in the marketplace, the idea of 'cultivation' is significant. It reminds us that ethics are developmental (not transcendental), that we need to learn to articulate and share what is ethical. It also suggests a need to foster the right environments and social structures to allow ethics to propagate. The cultivation of ethics requires greater depth and (shared) ownership, and also pertains to matters of the body and of habits (of how we embody ethical conduct). Beyond the abstract reports and codes of conduct on ethical practices and integrity, it is the complexities of cultivation that are critical to an account of integrity and intimacy, combined as a way of understanding how, practically and collaboratively, we attend appropriately to situations.

\section{Practicing Intimacy}

The pairing of 'intimacy' with 'integrity' leads away from a self-contained view of the subject to something closer to 'ensemble individualism' (Sampson 1988); or from independent to interdependent views of the self (Markus and Kitayama 1991), and importantly turns our attention to everyday examples of market exchange. The emphasis here is upon how we negotiate practices of both 'integrity' and 'intimacy' so as to (co-)exist to form an 'incident' or flow of individuals connecting with each other. As a matter of integrity, the shop assistant is expected to attend to all customers by equal measure. Yet, different rhythms can be at play. The experienced shopper, for example, may not wish to be disturbed, while a new customer could benefit from more attention, yet equally may feel uneasy to ask for help. There are various nuanced rhythms and interrelations in play. Reading the various signs, allowing for appropriate pauses, or entering into another's flow, are all subtle capabilities.

As a certain 'origin myth' of the interrelation of buyer and seller we can think back to Adam Smith's famous line from Wealth of Nations: 'It is not from the benevolence of the butcher, the brewer, or the baker, that we expect our dinner, but from their regard to their own interest. We address ourselves, not to their humanity but their self-love' (Smith 2009 b, p. 13). The everyday exchanges we can imagine between these traders of course gives rise to Smith's concept of the 'invisible hand', the unseen - and often suggested unobservable - market forces that ensure the supply and demand of goods achieve natural equilibrium. The point here is not to debate the relevant merits of the free market, but rather to take note of a specific 'unit of analysis' that is suggestive from Smith's evocation of 'the butcher, the brewer, or the baker'. In looking to contemporary scenarios, we can similarly think about the various sets of interests that are assumed in everyday, almost unobservable exchanges.
Imagine the following brief scenarios or vignettes as 'intimacies' of the marketplace:

A. Two worlds/tension/potential conflict Standing, waiting to pay, with the correct change in my hand, I must wait a moment as the person behind the counter jokes with their colleague about something that occurred the day before. They then turn to me to take my money, but only look sideways towards me as they continue to laugh with their colleague.

B. One world/relaxed/free to participate At the hair salon, as I am guided over to have my hair washed by one member of staff, another staff member moves quietly across the other side of the room. No words or gestures are exchanged, but just prior to being guided back to my seat in front of the mirror, someone has placed a drink before me.

C. Virtual world/convivial/scripted After some time on the phone, having been held in a queue listening to classical music, I am greeted cheerfully by name. The operator already has my details on screen as I entered in my account number before joining the queue. It turns out I cannot be helped today due to a software upgrade. I note my dissatisfaction to which the operator apologises again before asking 'is there anything else I can help you with today?'

These three scenes describe moments of exchange in specific situations and based upon distinct interpersonal rhythms. In the case of A, we witness rhythms that are out of sync. Ethically speaking, we might ask if it is so wrong for the person behind the counter to break from the monotony of work to joke with their colleague. Of course, for the customer, this fleeting, incidental moment can quickly feel like an eventful one. We become implicated in a situation that is not our own. We are subject to a configuration that we lack control over. Through the subtle, if unwitting power of the attendant, the flow of exchange is disrupted, and an unequal relationship is formed, the customer becomes the subject of disgruntlement. The case of $\mathrm{B}$, offers a much more fluid, harmonious example of integrating bodies. The two members of staff and the customer work in unison, or at least in sync. Their roles, their individual rhythms, are not blurred (the customer is still the customer). Yet, a single 'body' of movement and exchange is enacted, all undertaken without explicit direction. We might think of the image of a school of fish as they swim collectively, yet each allowing for individual 'tactful' spaces in-between. In the case of C, we might argue there is a similarly well-orchestrated flow of interaction, but which in this case still nonetheless leads to a less than satisfactory outcome. The failure of the computer system is a constraint on both sides-neither can achieve what they would expect in the situation. Yet, the operator, 
habitually trained to provide an established service, cannot help but ask whether they can help with anything else that day. It is a wholly redundant question in the situation, poorly judged and poorly timed.

The three scenarios can be thought of in terms of forms of 'practice', with reference to Bourdieu's (1977) account of practice and 'habitus'. The latter term is used to demarcate a site of practice between individual decision-making and supra-individual 'structures'; as well as referring to habitual or typical, cultivated conditions that relate particularly to the body. For Bourdieu practice is located in space and 'intrinsically defined by its tempo' (1977, p. 8), so being something 'that can be observed in three dimensions' (Jenkins 1992, p. 69). One of Bourdieu's key metaphors is the 'feel for the game', 'a mastery acquired by experience of the game, and one which works outside conscious control and discourse (in the way that, for instance, techniques of the body do)' (Bourdieu 1977, p. 61). This is a way of understanding anticipation. In tennis, for example, a common error is to play the ball (to lunge at it) rather than to play the bounce, which is to anticipate the ball in relation to swinging the racket. To relate this to the three cases above, it is only in the second example, at the hairdressers, that we can see the provider appropriately judging the situation. For any provider the 'shop' (or site of business, which can be a virtual space such as on the phone or a website etc.) has its own integrity, it is established as a setting. Yet, as soon as the customer enters into this space it becomes dynamic. In phenomenological terms, the 'shop' only ever comes into being through the activation of the customer and provider, which can take on many different modes depending on how this activation occurs. Despite the fact that a business appears from afar to be built upon repetitious exchanges, true integrity requires the provider to respond to each and every situation as it arises.

What is at stake is an aesthetic, spatial and temporal reading of one's relationship to a situation, or, in relation to the account given here in terms of both integrity and intimacy, i.e. one's response-ability to the situation. With regard to Bourdieu's notion of 'practice', this is to be considered for its fluidity and indeterminacy. We cannot abstractly define or predict practice, we can only know about it through doing. Bourdieu, for example, refers to 'necessary improvisation' (1977, p. 8), which, as Jenkins notes, brings us back to time, 'improvisation is the exploitation of pause, interval and indecision' (Jenkins 1992, p. 71). He goes on to argue: 'Social life, in all its complexity and variety, is not accomplished on the basis of rules, recipes and normative models. Imagine the impossibility, suggests Bourdieu, of having "on file" a rule or prescription of every conceivable situation which one might encounter in routine social life' (Jenkins 1992, p. 71). Of course, as with scenario $\mathrm{C}$, the call centre (and indeed any typical service scenario) is an obvious example of where an attempt is made to have 'on file', as a script, the prescription for every conceivable situation. From a business point of view we understand this as a matter of integrity, ensuring every individual user can be treated equally and to the same level of service. This is a laudable aim, and a reason why matters of integrity do indeed matter. Yet the scripting of any dialogue with a customer will inevitably fall short. There will be instances of 'deafness' simply because a script cannot predict everything a customer might say (or not say). In other words, the script is predicated on talking to the customer, not listening to them. The ubiquitous closing line of a service operator ('Is there anything else I can help you with?') is all too often the moment of revelation, when the conversation is exposed as a scripted dialogue. The effect can be particularly negative-as in scenario $\mathrm{C}$ - when the customer cannot be helped with an urgent problem, only to then be asked what becomes a futile question. Equally, it can ring hollow when the customer has been well served and is far from needing any further help. The customer, from an otherwise natural conversation, is brought back sharp to the script, exposing the fact that they are merely the customer. It would be easy to shift— to improvise-and respond to the fact that the customer is already satisfied by not asking a question, and simply acknowledge the (shared) outcome. Of course, this may not always be recognised as an ethical matter, except that knowing or sensing that one has been listened to makes a huge difference in how we understand the nature of the situation and our place within it.

Listening is indeed a form of ethics-it implies a whole series of considerations of and for the 'other' and the means of being together. The outsourcing of call centres overseas has further refined the rationalising and 'scripting' of relationships. Staff in call centres will receive extensive training to understand the cultural context to which they are servicing and will even adopt aliases in-keeping with the customer-base. This represents an intriguing and problematic case of the intertwining of integrity and intimacy. As Berkbigler and Dickson (2014) explain, there are varying levels of scripting (verbatim, emotional, computerized, and flexibility of scripting) designed to uphold the integrity of an exchange despite its geographic distance. In seeking to be 'true' to the context of the consumer there is the affectation of intimacy. For workers this has shown high levels of stress, particularly where scripting involves flexibility (Bigler and Dickson 2014) or, as termed here, anticipation and improvisation. Various businesses using overseas services have acknowledged many customers are not happy with the level of service. ${ }^{5}$ What is apparent is that it is hard

\footnotetext{
5 The UK's telecoms company, British Telecom (BT), for example, recently pledged $£ 80 \mathrm{~m}$, recruiting up to 1000 staff, to ensure at least $80 \%$ of its calls are answered from the UK (Davis 2016). The change in practice has come after Ofcom, the UK's telecoms regulator, iden-
} 
to simulate intimacy or proximity. As an ethical condition it is something that must be both genuinely expressed and felt. Listening, anticipating and improvising are all part of the means, the medium, through which a company maintains its relationship with the customer. By triangulating Kasulis' cultural philosophy, Bourdieu's notion of practice, and the broader Aristotlean view of culture as cultivation, there is a way in which we might nurture senses of intimacy. This refers to an on-going, lived process or practice, rather than a set of task-based rules that can be learnt.

In thinking with the above examples (scenarios $\mathrm{A}-\mathrm{C}$ ), specific aspects of intimacy can be drawn out. First, matters of proximity, i.e. how we respond to others through an awareness of space and the environment or situation in which we find ourselves. Second, of a more temporal nature is the ability to anticipate, to be aware of what can occur following our actions or that of others. Third, connecting with both spatial and temporal awareness is improvisation, an ability to respond appropriately in and through a given situation. The service provider will typically be cognisant of any given situation as a set of spatial and temporal connections. To frame this as a form of ethics, we can think of intimacy as being 'closely acquainted'; to place oneself in a position to find out about or be responsive to a person or situation. Furthermore, the position taken by this paper is to understand intimacy as bound up with the concept of integrity (with a view to expand how we practically engage with 'everyday' business ethics). Rather than simply tempering what we mean by integrity (when defined as substantive moral virtues, as discussed earlier in this paper), the introduction of intimacy is to understand how we actually perform integrity; and crucially it is not rooted in the individual, but always as an exchange between others. In this way, integrity needs its intimacy: we need to find the means to be mindful and responsive to the incidental if we are to be eventful in our actions and interactions. Overall, then, in thinking through how we interact in-and indeed practice-social-business exchanges we need to consider an Integrity-Intimacy Approach, which is to find ways to properly associate principles with practices of being together.

\section{Integrity-Intimacy Approach}

Smith's famous line of the 'benevolence' of the butcher, the brewer, or the baker, is all too often read as the symptom of mere calculation. However, this is to assume an atomistic account of the individuals involved in the exchange.

\section{Footnote 5 (continued)}

tified BT as one of the worst companies in the industry; it can be considered then a strategy towards 'intimacy' in order to uphold integrity.
The relationship is defined as one of the equidistance of bodies/subjects, so suggestive of a relation to one another, not a relation between each other. By contrast, in Kasulis' Intimacy or Integrity (2002), the difference is expressed as either a 'belonging to' or a 'belonging with'. He gives an example of having one's wallet stolen, which contains both money and treasured family photos. While the money belonged to you, he notes, the 'pictures belonged with you not to you. In taking the photos, the thief stole part of your self, not merely something external like money over which you held temporary title' (p. 23). In setting out a contrast between integrity (as befitting the account given in this paper) and intimacy, Kasulis uses this sense of 'belongingwith' to identify a difference between external relations (associated with integrity) and internal relations (associated with intimacy): 'In an external relation, the relatents (the things in relation to each other) exist independently. [...] In an internal relation, by contrast, it is part of the essential nature of the relatents that they are connected as they are; they are interdependent, not independent, entities' (p. 36). Put simply, we might say integrity is a quality that pertains to ourselves, while intimacy is never our own.

As with the earlier image of a school of fish, elements can be distinct in themselves, yet hold together as a single form. A key aspect of intimacy is this notion of 'belongingwith', 'the self and other belong together in a way that does not sharply distinguish the two' (Kasulis 2002, p. 32). This establishes an important underlying principle. If we read the situation of the butcher and customer as a matter of intimacy (even if only temporarily for the duration of an exchange), we immediately change our perspective on the unit of analysis, which becomes a singular, or at least joined-up choreographed 'body', rather than two distinct individuals; we cannot separate one from the other. In line with Smith's (2009a) thinking on the role of the conscience and sympathy, which leads to his theory of moral sentiment - or even sentiencethere are two related aspects to intimacy: empathetic imagination and embodiment. The notion of 'belonging-with' suggests of an ability to step outside of ourselves, and/or to remain a part of something. This is evident with scenario B as a whole or integrated form; it is unbalanced in scenario A where the service provider chooses to belong more with their colleague than their customer; and in scenario $\mathrm{C}$ it is arguably that 'belonging-with' is over-played or simulated. What is at stake is a set of rhythms for engagement and tacit knowledge. As Kasulis points out 'knowledge is generally transmitted or taught in a nondiscursive way' $(2002$, p. 40), but that it plays an important role in 'the development of morality, technical skill with a tool, and aesthetic sensitivity. [...T]here is not only empirical observation and logical reasoning; there is also an emotive identification with a person or the use of an object' (2002, p. 41). As an embodiment of knowledge, this can be said to relate to the Aristotlean 
'cultivation' of characteristics that Solomon refers to, which exist over time, can be shared in various indirect ways, and which operate in as much implicit as in explicit ways.

Ideas about aesthetic sensitivity and the body have had a clear impact on the retail sector, particularly where businesses need to rethink the purpose and status of face-toface interaction following the rise of Internet services. The ability to book our own tailored travel arrangements online has inevitably impacted on travel agents. One response has been to design shops to appear more like a lounge, to allow customers to effectively book their travel on computers as they might at home. The removal of counters and staffoperated computers creates a more horizontal relationship between those serving and being served, effectively altering the ethicality of exchange. It is no longer about attending to, but being with another. Surprisingly, the banking sector is also going in a similar direction. While we might associate notions of traditionalism and hierarchy with those ordained to look after our money, the dominance of Internet-based services is leading this sector to re-stage its operations. Milligan (2016) notes that while we typically hear of the widespread closure of branches, in 2017 three British banks were due to open more branches than they would close. These new and/or refurbished branches, however, tend to look more like cafes than banks. Similarly, in the US, Capital One, which launched as a digital-only bank, has thirteen banking 'cafes' across the country. The aesthetic arrangements and handling or staging of environments in which we come together for exchange can in themselves help cultivate certain habits, and the embodiment of proximity, anticipation, and improvisation. Entering into a bank for a coffee, for example, immediately establishes a different kind of engagement. The customer is likely to sit down, to be receptive to dialogue and to take more time over the purpose of their visit.

Kasulis also refers to intimacy as esoteric, by which he means that like tacit knowledge, intimacy is not immediately obvious (or communicable) and must be gained over a period of time and through learning - and even then, crucially, we do not necessarily have clear access to an understanding, it is just something we can do (or at least we are 'cultivated' to able to do). He gives the example of a sportsperson able to perform a feat of great skill and timing, without necessarily being able to explain or even describe it. Underlying all is a final key characteristic of intimacy: it is personal, not public, yet it is objective. Kasulis gives various instructive examples to explain this point. For example, a physician is able to discern a particular diagnosis when viewing an X-ray, while the untrained eye would see only cloudy shapes. The $\mathrm{X}$-ray offers empirical evidence, yet this is not something that can be read by anyone. Similarly, a judge at a gymnastics event is able to read the various elements of a routine to give a fair score. There is a speed and agility to this assessment (that matches the routine of the gymnast themselves).
'In such sporting events as gymnastics, figure-skating, surfing, and diving', Kasulis notes, 'the score for the degree-ofdifficulty of a performance is usually a publicly verifiable judgement based on the empirically observable sequence of movements. Given a detailed rulebook and a videotape of the performance, even a novice to the field should be able to determine such a score' (2002, p. 34). The idea here of a public, empirically verifiable judgment makes for an important principle to be transposed to the business context. While there is arguably a subtle 'art' to staging the business setting and guiding for the right kinds of interaction with customers, placing greater scrutiny and investment upon such matters should yield greater expertise. I.e. intimacy, in the sense identified here, is not merely instinctive, but rather defines a form of practice, for which we can train, display and verify.

If we think back to the scenarios outlined above, A (standing before the cashier), B (waited on at the hair salon) and C (engaging with a phone operator), in which the failures and success of intimacy are evident, we can argue (in line with Kasulis' account of the objectivity of intimacy) that the intimate knowledge of scenario B could find its equivalence in scenarios A and C. In the case of A, for example, the cashier only needs to be aware of the relative proximity of the customer and to better anticipate actions (which may or may not allow time for an aside with their colleague, depending on the flow of engagements). Similarly, the operator described in scenario $\mathrm{C}$, while needing to deal with an obviously difficult situation in which the computer is not working, needs to better anticipate and empathise with the callers frustration. This requires improvisation, which again, is to be attuned to the lived rhythms of the situation. Nonetheless, these are often difficult and subtle adjustments that are required. It involves accessing private, imaginative, embodied, and esoteric forms of knowledge. Yet, importantly, these knowledges do have an empirical basis. If, for example, we slow down the videotape of the gymnast we can locate the same empirically observable sequence of movements that the expert judge is able to read in real time. This account of intimacy implies the need for long-term engagement and exposure. It is a form of learning that goes beyond discursive knowledge exchange, thus contrasting with integrity, which can be communicated more directly. Integrity is exchangeable, making it the dominant 'currency' for how we assess business ethics, while it is through its intimate cultivation that integrity sustains.

Kasulis' (2002) account of the differing orientations of integrity and intimacy suggests of one being made dominant over the other, which he describes in terms of a cultural ideology, or, to use his metaphor an 'operating system' on which more complex 'programs' can run. In this sense, integrity and intimacy represent philosophical systems that determine how we tend to think about the world, ourselves and society (p. 71). This leads to the suggestion that the 
differing emphasis of integrity/intimacy is culturally specific (though he argues strongly against cultural essentialism). The key point is that cultures-and more particularly subcultures and institutional groups (such as managers or employees) — can foreground different principles, 'one can at least imagine a culture that places a primary, rather than secondary, value on the enhancement of intimacy' (p. 51). Note, however, the position taken by this paper implies a critique of Kasulis' binary opposition, since the argument made is that we need both. Considered deconstructively, neither holds without the other. In thinking practically about managing the marketplace for the better, we need to observe the day-to-day, to consider mood, tone, and habits, as much as there is a need to audit for principles of practice. In seeking to resolve ethical tensions, or indeed in seeking to prevent them in the first place, it is necessary to understand what is being exchanged, and crucially how things are exchanged. It is important to acknowledge the 'response-abilities' of all actors and mechanisms involved in the exchange. It is worth noting the ubiquity of social media has led to a more collective or 'horizontal' nature of marketing, which typically underscores how it is counter-productive to deceive or pressure the consumer. In terms similar to Kasulis, Abela and Murphy (2008) suggest a shift is required to frame the business to customer relationship not as 'doing to' (with integrity) but as 'doing with' (as both integrity and intimacy). Significantly, the inclusion of intimacy as a measurable or at least observable dimension, while undoubtedly subtle, offers further import. It helps us return to the occasion when people consider what others feel; and if only to be situated with regard to the kinds of incidences of empathy that Smith drew our attention to centuries before.

\section{Research and Managerial Implications}

The foregoing account of the intimacies of integrity in everyday market exchanges can lead us to three inter-related research and managerial implications. In the first instance, it is incumbent on businesses (and their managers) to pay ever-greater attention to the spatial and temporal dynamics and proximities of the exchanges they invoke. This relates to the physical environment (i.e. how consumer spaces are designed for interactions with customers, rather than simply transactions) also virtual environments or interactions (e.g. online customer help), and gestural forms of exchange that allow a customer to feel part of a wider whole. There is a good deal of interest in the design and operational management of consumer spaces, but typically the focus is on aspects of efficiency in service delivery, the communication of corporate identity or brand, and the offer of certain moods and atmosphere. The suggestion here is the need to combine this understanding of spatial/temporal designs with maintaining integrity through intimacies of exchange. I.e. to develop a more granular analysis of situations.

The need to get in closer to situations of exchange presents new methodological considerations. We cannot simply describe and/or reframe matters of integrity through principles. It is necessary to understand how situations unfold intimately, which is to engage empirically with a different unit of analysis, which, fundamentally, we can relate to in terms of 'rhythms'. While beyond the purview of this current article, it is worth noting that the relatively recent posthumous publication (in English) of Henri Lefebvre's Rhythmanalysis (2013) marks a wider interest in recent critical theory. The European research network, Rhuthmos, ${ }^{6}$ makes a strong case for the pertinence of rhythmanalysis at our current conjuncture. In 'A Short History of Rhythm Theory Since the 1970s' (2011), for example, Michon demonstrates how questions of rhythm were always present in twentieth century European philosophy (and dating back to ancient philosophy), but have come back much more forcefully today. The argument made is that on the one hand the force of post-structuralist theory is much less significant today (if only because the critical ideas have been readily subsumed into a more critically aware culture); while on the other hand, the previously implicit interest in rhythm has become much more relevant in the context of our rapidly changing and data-driven society. The genre of critical management studies has been important in bringing across to business the key ideas of poststructuralist critical theory, however, these literatures tend to work with broad-brush accounts and remain within the 'grand narratives' of Marxism, feminism, institutional discourse and anti-foundationalism more generally. The suggestions made here regarding 'practices of intimacy' can be taken to implicate a new level of critical reading and its application in the context of business. Certainly, rhythm analysis represents a fertile avenue for future, interdisciplinary research.

Finally, on a practical level, the import of an 'intimate' analysis (through new methodological approaches) can enable businesses to rethink how they design and cultivate their relationship with stakeholders. It is possible, for example, to re-visit training, to get away from rule-based understanding in favour of improvisation, with the aim to be responsive as much as responsible, by which we can understand a need to extend our 'response-abilities' (so for example to be able to respond to a situation by attending to proximities). Crucially, such training cannot be delivered as goal-orientated. In the Aristotelian tradition, intimacies of integrity require the

\footnotetext{
${ }^{6}$ The European research network Rhuthmos (http://rhuthmos.eu), under the direction of Pascal Michon, offers an international and transdisciplinary research platform on rhythms in the sciences, philosophies and the arts.
} 
cultivation of community rather than organisational cohesion. The pursuit of the 'good life' requires that there is a form of living to begin with. It is at this level that intimacy broaches a way of thinking and acting, which we can observe and be trained for, but which is a means towards ethical engagement rather than ethical content itself. The underlying point for businesses is to understand integrity not merely as a measuring stick, but as a dynamic process that takes place through the medium of exchange. The implication for managers is the need to pay greater attention on an everyday basis to the medium of exchange. Painter-Morland makes reference to Nietzsche's account of 'responsibility-debt', which often emerges through the reactive forces found in training and selection processes. This is to refer to a type of responsibility that 'belongs to a role, or arises out of guilt' (2011, p. 90). The idea of engendering a greater sense of 'intimacy' through training ought to go beyond any mechanistic, didactic approach to train for intimacy. It requires a more extended and interactive or shared process of cultivation. Nonetheless, a nuanced and relational reading of situations can lead to a more adept choreographing of the customer experience, offering greater flexibility through employee training, and more generally the cultivation of 'response-abilities'.

\section{Conclusion}

The paper addresses the problem of pursuing good practice in business ethics by thinking about the 'how' or 'medium' of our everyday actions within the context of commercial exchange. Relying only on the idea of the integrity of a system, and the corresponding research and investment related to the pursuit of integrity, is arguably only to lead to failure (or to varying forms of so-called greenwash reporting). Through various examples of the business setting, it is argued that everyday considerations of proximity and staging, along with our abilities (and willingness) to anticipate and improvise within situations, can change the nature of outcomes. Crucially, paying attention to outcomes as a comprehensive, cultivated process, leads us to question how we achieve our actions. In this regard, intimacy as a critical concept is important to combine with any account of, or aspiration towards, integrity.

Traced in relation to critical management literatures, intimacy can be seen to add, pragmatically, to post-structuralist accounts of business, in this case to open up ideas of responsiveness or 'response-abilities', as opposed simply to responsibilities. The term provides a means of identifying practical, if subtle, attributes and practices at an interpersonal level, to relate always to the interaction with others. Even in a simple setting such as a café, service is not simply the correct serving of a drink (as a matter of integrity), but the serving of 'service' itself. There is a manner of purveying the drink to another that is inherently an ethical exchange; an exchange of what it means to live appropriately, to live a good life not just for oneself but necessarily with others. From the Ancient Greek ethikos, we derive the word ethos, which refers to habit and custom. Intimacy allows us to think and look critically and empirically at the level of habit and the everyday, which we can then combine with an understanding of integrity as ethical systems that businesses seek to 'employ'.

The idea of intimacy in business exchanges, as characterised in this paper, might suggest of the need for higher levels of staffing and/or higher quality training. This is too simple an equation, but certainly it responds in a particular way to the tension between efficiency and effectiveness in business. It can be efficient to reduce staff, but in the long run this can impact on effectiveness. Equally, of course, having too many staff in a situation ('too many cooks') may impede effectiveness. As outlined in the aforementioned implications, there are various spatial and temporal considerations that can be made of situations of exchange, as well as differing rhythms to which we can pay attention, and which can be observed if we take the time to do so. The point about intimacy-as with the case of watching the gymnast-is that we can seek to better observe how a situation operates or unfolds, and so then to discern appropriate requirements. To apply a greater sense of intimacy in regard to integrity (achieved through matters of proximity, anticipation and improvisation) leads us towards a shift in ethical thinking. Businesses need to align with others, to make their customers' (and other stakeholders') issues become their own, so as to be shared. It is important, then, to respond to and/ or pre-empt problems, which requires being as responsive to a situation as those whom we wish to be responsible towards. Thus, being able to anticipate, accommodate and respond to others (to adopt relational concerns) is a response-ability we ought to pursue, as part of the pursuit of integrity.

Acknowledgements The author would like to thank Patrick Murphy for his comments on an early version of the paper, as well as the Journal's section editor Christopher Michaelson and anonymous reviewers for their insightful feedback.

\section{Compliance with Ethical Standards}

Conflict of interest The author declares that she has no conflict of interest.

Ethical Approval This article does not contain any studies with human participants performed by the author.

Open Access This article is distributed under the terms of the Creative Commons Attribution 4.0 International License (http://creativeco mmons.org/licenses/by/4.0/), which permits unrestricted use, distribution, and reproduction in any medium, provided you give appropriate credit to the original author(s) and the source, provide a link to the Creative Commons license, and indicate if changes were made. 


\section{References}

Abela, A. V., \& Murphy, P. E. (2008). Marketing with integrity: Ethics and the service-dominant logic for marketing. Journal of the Academy of Marketing Science, 36(1), 39-53.

Audi, R., \& Murphy, P. E. (2006). The many faces of integrity. Business Ethics Quarterly, 16(1), 3-21.

Bauman, D. C. (2013). Leadership and the three faces of integrity. The Leadership Quarterly, 24, 414-426.

Berkbigler, E., \& Dickson, K. E. (2014). Required scripting and work stress in the call center environment: A preliminary exploration. Journal of Organizational Culture, Communications and Conflict, 18(1), 233-255.

Bourdieu, P. (1977). Outline of a theory of practice (Richard Nice, Trans.). Cambridge: Cambridge University Press.

Cage, J. (1957). Silence. Cambridge, MA: MIT Press.

Cunliffe, A. L., \& Eriksen, M. (2011). Relational leadership. Human Relations, 64(11), 1425-1449.

Dalla Costa, J. (1998). The ethical imperative. Reading: Addison-Wesley.

Davis, R. (2016). BT hires 1,000 UK staff after complaints over Indian call centres. Guardian, Monday 18 January, 2016. Retrieved January 21,2017 , from https://www.theguardian.com/business/2016/ jan/18/bt-hires-1000-uk-staff-after-complaints-indian-call-centres.

De George, R. T. (1993). Competing with integrity in international business. New York: Oxford University Press.

Fukukawa, K., \& Teramoto, Y. (2009). Understanding Japanese CSR: The reflections of managers in the field of global operations. Journal of Business Ethics, 85, 133-146.

Gostick, A., \& Telford, D. (2003). The integrity advantage. Salt Lake City: Gibbs Smith.

Jenkins, R. (1992). Pierre Bourdieu. London: Routledge.

Jensen, M. C. (2009). Integrity: Without it nothing works. Rotman Magazine, 16-20.

Kasulis, T. P. (2002). Intimacy or integrity: Philosophy and cultural difference. Honolulu: University of Hawaii Press.

Krylova, K. O., Jolly, P. M., \& Phillips, J. S. (2017). Followers' moral judgments and leaders' integrity-based transgressions: A synthesis of literatures. The Leadership Quarterly, 28, 195-209.

Lizardo, O. (2004). The cognitive origins of Bourdieu's habitus. Journal for the Theory of Social Behaviour, 34(4), 375-448.
Maak, T. (2008). Undivided corporate responsibility: Toward a theory of corporate integrity. Journal of Business Ethics, 82, 353-368.

Markus, H. R., \& Kitayama, S. (1991). Culture and the self: Implications for cognition, emotion, and motivation. Psychological Review, 98(2), 224-253.

Michon, P. (2011) A short history of rhythm theory since the 1970s, Rhuthmos, Retrieved December 6, 2011 from http://rhuthmos.eu/ spip.php?article462.

Milligan, B. (2016) Branch banking takes on a new fragrance for 2017. BBC News. Retrieved January 21, 2016 from http://www.bbc. co.uk/news/business-38380465.

Monga, M. (2016). Integrity and its antecedent: A unified conceptual framework of integrity. The Journal of Developing Areas, 50(5), 415-421.

Paine, L. S. (1997). Integrity. In P. H. Werhane \& R. E. Freeman (Eds.), The blackwell encyclopedic dictionary of business ethics (pp. 335-336). Oxford: Blackwell Publishers Ltd.

Painter-Morland, M. (2011). Rethinking responsible agency in corporation: Perspectives from Deleuze and Guattari. Journal of Business Ethics, 101, 83-95.

Rancière, J. (2004). The politics of aesthetics: The distribution of the sensible (Rockhill Gabriel, Trans.). London: Continuum.

Sampson, E. E. (1988). The debate on individualism: Indigenous psychologies of the individual and their role in personal and societal functioning. American Psychologist, 43(1), 15-22.

Selznick, P. (1994). The moral commonwealth: Social theory and the promise of community. Berkeley: University of California Press.

Smith, A. (2009a[1759]). The theory of moral sentiments. New York: Penguin Group.

Smith, A. (2009b[1776]). An inquiry into the nature and causes of the wealth of nations. Overland Park: Digireads.com Publishing.

Solomon, R. C. (1992). Ethics and excellence: Cooperation and integrity in business. New York: Oxford University Press.

Vargo, S. L., \& Lusch, R. F. (2008). Service-dominant logic: Continuing the evolution. Journal of the Academy of Marketing Science, $36(1), 1-10$

Zauderer, D. G. (1992). Integrity: An essential executive quality. In Business forum (pp. 12-16). Los Angeles: Califomia State University. 